\title{
The Regular Part of a Semigroup of Full Transformations with Restricted Range: Maximal Inverse Subsemigroups and Maximal Regular Subsemigroups of Its Ideals
}

\author{
Worachead Sommanee (iD) \\ Department of Mathematics and Statistics, Faculty of Science and Technology, Chiang Mai Rajabhat University, \\ Chiang Mai 50300, Thailand
}

Correspondence should be addressed to Worachead Sommanee; worachead_som@cmru.ac.th

Received 9 March 2018; Revised 25 March 2018; Accepted 1 April 2018; Published 7 May 2018

Academic Editor: Pentti Haukkanen

Copyright (C) 2018 Worachead Sommanee. This is an open access article distributed under the Creative Commons Attribution License, which permits unrestricted use, distribution, and reproduction in any medium, provided the original work is properly cited.

Let $T(X)$ be the full transformation semigroup on a set $X$. For a fixed nonempty subset $Y$ of a set $X$, let $T(X, Y)$ be the semigroup consisting of all full transformations from $X$ into $Y$. In a paper published in 2008, Sanwong and Sommanee proved that the set $F(X, Y)=\{\alpha \in T(X, Y): X \alpha=Y \alpha\}$ is the largest regular subsemigroup of $T(X, Y)$. In this paper, we describe the maximal inverse subsemigroups of $F(X, Y)$ and completely determine all the maximal regular subsemigroups of its ideals.

\section{Introduction}

Let $T(X)$ be the set of all full transformations from a nonempty set $X$ into itself. It is well-known that $T(X)$ is a regular semigroup under composition of functions; see [1, p. 63]. Moreover, every semigroup can be embedded in $T(X)$ for some nonempty set $X$; see [1, p. 7]. In [2, 3 ], Schein posed the problem to determine all maximal inverse subsemigroups of $T(X)$; this problem is still unsolved. In 1976, Nichols [4] characterized one class of maximal inverse subsemigroup of $T(X)$. Later in 1978, Reilly [5] has generalized Nichols construction and obtained a much wider class of maximal inverse subsemigroups of $T(X)$. In 1999, Yang [6] described all of the maximal inverse subsemigroups of the finite symmetric inverse semigroup. Later in 2001, Yang [7] obtained the maximal subsemigroups of the finite singular transformation semigroups. In 2002, You [8] determined all the maximal regular subsemigroups of all ideals of the finite full transformation semigroup. In 2004, H. B. Yang and X. L. Yang [9] completely described the maximal subsemigroups of ideals of the finite full transformation semigroup. In 2014, Zhao et al. [10] showed that any maximal regular subsemigroup of ideals of the finite full transformation semigroup is idempotent generated. After that in 2015, East et al. [11] classified the maximal subsemigroups of $T(X)$ when $X$ is an infinite set containing certain subgroups of the symmetric group on $X$.

Let $Y$ be a fixed nonempty subset of $X$ and let $T(X, Y)$ be the subsemigroup of $T(X)$ of all elements with ranges contained in $Y$. In 1975, Symons [12] introduced and studied the semigroup $T(X, Y)$. He described all the automorphisms of $T(X, Y)$ and also determined the isomorphism theorem for two semigroups of type $T(X, Y)$. In 2005, Nenthein et al. [13] characterized the regular elements of $T(X, Y)$. In general, $T(X, Y)$ is not a regular semigroup. In 2008, Sanwong and Sommanee [14] defined

$$
F(X, Y)=\{\alpha \in T(X, Y): X \alpha=Y \alpha\}
$$

and showed that $F(X, Y)$ is the largest regular subsemigroup of $T(X, Y)$. Obviously, $F(X, Y)=T(X)$ when $X=Y$. Hence, we may regard $F(X, Y)$ as a generalization of $T(X)$. The authors also characterized Green's relations on $T(X, Y)$ and gave one class of maximal inverse subsemigroups of $T(X, Y)$. Later in 2009, Sanwong et al. [15] described all maximal and minimal congruences on $T(X, Y)$. In 2011, Mendes-Gonçalves and Sullivan [16] obtained all the ideals of $T(X, Y)$. In the same year, Sanwong [17] described Green's relations and ideals and all maximal regular subsemigroups of $F(X, Y)$. The author also proved that every regular semigroup $S$ can 
be embedded in $F\left(S^{1}, S\right)$. After that, in 2013, Sommanee and Sanwong [18] computed the rank of $F(X, Y)$ when $X$ is a finite set. They also obtained the rank and the idempotent rank of its ideals when $X$ is finite. In 2014, Fernandes and Sanwong [19] calculated the rank of $T(X, Y)$ when $X$ is finite. In 2016, L. Sun and J. Sun [20] characterised the natural partial order on $T(X, Y)$. In the same year, Tinpun and Koppitz [21] determined the relative rank of $T(X, Y)$ modulo the semigroup $S(X, Y)$ of all extensions of the bijections on $Y$ when $X$ is finite.

Let $X$ be a chain and OT $(X)$ the full order-preserving transformation semigroup on $X$. In 2011, Dimitrova and Koppitz [22] characterized the maximal regular subsemigroups of ideals of OT $(X)$ when $X$ is a finite chain. In 2015, Sommanee and Sanwong [23] investigated the regularity and Green's relations of the order-preserving transformation semigroup

$$
\begin{aligned}
\mathrm{OF}(X, Y) & =F(X, Y) \cap \mathrm{OT}(X) \\
& =\{\alpha \in F(X, Y): \alpha \text { is order-preserving }\} .
\end{aligned}
$$

They also proved that $O F(X, Y)$ is idempotent generated when $Y$ is finite.

Let $V$ be a vector space and let $T(V)$ denote the linear transformation semigroup from $V$ into $V$. For a fixed subspace $W$ of $V$, let $T(V, W)$ be the semigroup consisting of all linear transformations from $V$ into $W$. Recently in 2017, Sommanee and Sangkhanan [24] determined all the maximal regular subsemigroups of the semigroup

$$
Q=\{\alpha \in T(V, W): V \alpha=W \alpha\},
$$

when $W$ is a finite dimensional subspace of $V$ over a finite field.

In this paper, we describe the maximal inverse subsemigroups of $F(X, Y)$ and completely determine all the maximal regular subsemigroups of its ideals.

\section{Preliminaries and Notations}

For all undefined notions, the reader is referred to [1].

For any set $A,|A|$ means the cardinality of the set $A$. If $A$ is a subset of a semigroup $S$, then $\langle A\rangle$ denotes the subsemigroup of $S$ generated by $A$. An element $e$ of a semigroup $S$ is called idempotent if $e^{2}=e$. If $U$ is a subset of a semigroup $S$, then $E(U)$ denotes the set of all idempotents in the set $U$. An element $a$ of a semigroup $S$ is called regular if there exists $x \in S$ such that $a=$ axa. The semigroup $S$ is called regular if all its elements are regular. If $a$ is an element of a semigroup $S$, we say that $a^{\prime}$ is an inverse of $a$ if $a=a a^{\prime} a$ and $a^{\prime}=a^{\prime} a a^{\prime}$. We denote the set of inverses of an element $a$ by $V(a)$. A semigroup $S$ is called an inverse semigroup if every element $a$ in $S$ has a unique inverse $a^{-1}$ in $S$; it is equivalent to Sbeing regular and idempotent elements commute. A proper (regular, inverse) subsemigroup $M$ of a semigroup $S$ is a maximal (regular, inverse) subsemigroup of $S$ if whenever $M \subseteq T \subseteq S$ for some a (regular, inverse) subsemigroup $T$ of $S$, then $M=T$ or $T=S$.

The Green's relations $\mathscr{L}, \mathscr{R}, \mathscr{H}, \mathscr{D}$, and $\mathscr{J}$ on a semigroup $S$ are defined as follows. For $a, b \in S$,
(1) $a \mathscr{L} b$ if and only if $S^{1} a=S^{1} b$;

(2) $a \mathscr{R} b$ if and only if $a S^{1}=b S^{1}$;

(3) $a \mathscr{J} b$ if and only if $S^{1} a S^{1}=S^{1} b S^{1}$;

(4) $\mathscr{H}=\mathscr{L} \cap \mathscr{R}$ and $\mathscr{D}=\mathscr{L} \circ \mathscr{R}$.

For each $a \in S$, we denote $\mathscr{L}$-class, $\mathscr{R}$-class, $\mathscr{H}$-class, $\mathscr{D}$ class, and $\mathscr{J}$-class containing $a$ by $L_{a}, R_{a}, H_{a}, D_{a}$, and $J_{a}$, respectively.

Lemma 1 (see [5, Lemma 1]). Let $M$ be a maximal inverse subsemigroup of a semigroup $S$. If $M$ contains a minimum idempotent $e$, then $H_{e} \subseteq M$.

Lemma 2. Let $S$ be any inverse semigroup. If e is a minimum idempotent in $S$, then ea $=$ ae for all $a \in S$.

Proof. Assume that $e$ is a minimum idempotent in $S$. Then $e \leq f$ for all $f \in E(S)$; that is, $e=e f=f e$. Let $a \in S$. Then $a e a^{-1}, a^{-1} e a \in E(S)$ and $e a=e\left(a e a^{-1}\right) a=\left(a e a^{-1}\right) e a=$ $a\left(e a^{-1} e a\right)=a e$.

Throughout the paper we assume that $Y$ is a nonempty subset of a set $X$. Let $T(X, Y)=\{\alpha \in T(X): X \alpha \subseteq Y\}$, where $X \alpha$ denotes the image of $\alpha$. Define

$$
F(X, Y)=\{\alpha \in T(X, Y): X \alpha=Y \alpha\}
$$

It is known that $F(X, Y)$ is the largest regular subsemigroup of $T(X, Y)$.

If $\alpha \in T(X)$ and $x \in X$, then the image of $x$ under $\alpha$ is written as $x \alpha$. The set of all inverse images of $x$ under $\alpha$ is denoted by $x \alpha^{-1}$.

Lemma 3 (see [17, Lemma 1]). Let $\alpha \in T(X, Y)$. Then $\alpha \in$ $F(X, Y)$ if and only if $a \alpha^{-1} \cap Y \neq \emptyset$ for all $a \in X \alpha$.

In [17], the author gave a complete description of Green's relations on $F(X, Y)$ as the following lemma.

Lemma 4. Let $\alpha, \beta \in F(X, Y)$. Then

(1) $\alpha \mathscr{L} \beta$ if and only if $X \alpha=X \beta$;

(2) $\alpha \mathscr{R} \beta$ if and only if $\pi_{\alpha}=\pi_{\beta}$, where $\pi_{\alpha}=\left\{x \alpha^{-1}: x \in\right.$ $X \alpha\}$

(3) $\alpha \mathscr{D} \beta$ if and only if $|X \alpha|=|X \beta|$;

(4) $\mathscr{D}=\mathscr{J}$.

In addition, $\alpha \mathscr{H} \beta$ if and only if $X \alpha=X \beta$ and $\pi_{\alpha}=\pi_{\beta}$.

Lemma 5. $F(X, Y)$ is an inverse semigroup if and only if $|Y|=$ 1.

Proof. If $|Y|=1$, then $|F(X, Y)|=1$ and so it is inverse. Conversely, assume that $F(X, Y)$ is an inverse semigroup. Let $a$ and $b$ be elements in $Y$. Then there exist constant maps $X_{a}$ and $X_{b}$ with ranges $\{a\}$ and $\{b\}$, respectively. Thus they are idempotents in $F(X, Y)$ such that $X_{a}=X_{b} X_{a}=X_{a} X_{b}=X_{b}$; it follows that $a=b$. Hence, $|Y|=1$. 
Proposition 6. $M$ is a maximal inverse subsemigroup of $T(X, Y)$ if and only if $M$ is a maximal inverse subsemigroup of $F(X, Y)$.

Proof. One direction is clear. Indeed, each inverse subsemigroup of $T(X, Y)$ is contained in $F(X, Y)$, the largest regular subsemigroup of $T(X, Y)$. Conversely, assume that $M$ is a maximal inverse subsemigroup of $F(X, Y)$. Then $M$ is an inverse subsemigroup of $T(X, Y)$. Let $V$ be an inverse subsemigroup of $T(X, Y)$ such that $M \subseteq V \varsubsetneqq T(X, Y)$. Since $F(X, Y)$ is the largest regular subsemigroup of $T(X, Y)$, it follows that $M \subseteq V \subseteq F(X, Y)$. We get that $M=V$ or $V=F(X, Y)$ by the maximality of $M$ in $F(X, Y)$. If $F(X, Y)=$ $V$, then $F(X, Y)$ is an inverse semigroup and so $|Y|=1$ by Lemma 5; this implies that $T(X, Y)=F(X, Y)$. Therefore, $M$ is a maximal inverse subsemigroup of $T(X, Y)$.

For each $a \in Y$, let

$$
\begin{aligned}
F_{a} & =\{\alpha \in F(X, Y): a \alpha \\
& \left.=a, \alpha \text { is injective on } X \backslash a \alpha^{-1}\right\} .
\end{aligned}
$$

The authors [14] showed that $F_{a}$ is a maximal inverse subsemigroup of $T(X, Y)$. Thus by Proposition 6 , we have that $F_{a}$ is also a maximal inverse subsemigroup of $F(X, Y)$.

\section{Maximal Inverse Subsemigroups}

In this section, we write $G(A)$ for the permutation group on a set $A$.

Let $e$ be any idempotent in $T(X)$. In 1978, Reilly defined $I_{e}$ to be the set of those elements $\alpha \in T(X)$ which satisfy the following three conditions:

(1) The restriction of $\alpha$ to $X e$ is a permutation of $X e$; that is, $\left.\alpha\right|_{X e} \in G(X e)$.

(2) For $x \in X$, if $x \alpha \notin X e$, then $\left|(x \alpha) \alpha^{-1}\right|=1$.

(3) $\alpha e=e \alpha$.

Lemma 7 (see [5, Theorem 2]). Let e be an idempotent in $T(X)$ and let $I_{e}$ be defined as above. Then $I_{e}$ is a maximal inverse subsemigroup of $T(X)$ with minimum idempotent $e$.

Notice that if $e$ is a constant map with range $\{a\} \subseteq X$, then $I_{e}=I_{a}$, where

$$
I_{a}=\left\{\alpha \in T(X): a \alpha=a, \alpha \text { is injective on } X \backslash a \alpha^{-1}\right\} ;
$$

see $[4,5]$ for more details.

Now, let $e$ be any idempotent in $F(X, Y)$ and define

$$
F_{e}=I_{e} \cap F(X, Y) .
$$

Since $e \in I_{e} \cap F(X, Y)=F_{e}$, we obtain $F_{e} \neq \emptyset$ and so $F_{e}$ is a subsemigroup of $F(X, Y)$. We note that if $X=Y$, then $F(X, Y)=T(X)$ and $F_{e}=I_{e} \cap F(X, Y)=I_{e} \cap T(X)=I_{e}$.

Here, we aim to prove that $F_{e}$ is the maximal inverse subsemigroups of $F(X, Y)$ with minimum idempotent $e$.

To prove our main result we need the following five lemmas.
Lemma 8. Let $e$ be any idempotent in $F(X, Y)$ and $\alpha \epsilon$ $F(X, Y)$. Then $\alpha \in F_{e}$ if and only if

(1) $\left.\alpha\right|_{X e} \in G(X e)$,

(2) for $y \in Y$, if $y \alpha \notin X e$, then $\left|(y \alpha) \alpha^{-1}\right|=1$,

(3) $\alpha e=e \alpha$.

Proof. One direction is clear. Conversely, assume that conditions (1), (2), and (3) hold. We prove that $\alpha \in I_{e}$. Suppose that $x \in X$ and $x \alpha \notin X e$. Since $\alpha \in F(X, Y)$, it follows that $x \alpha=y \alpha$ for some $y \in Y$. By condition (2), we get that $\left|(y \alpha) \alpha^{-1}\right|=1$. Thus $\left|(x \alpha) \alpha^{-1}\right|=1$ and so $\alpha \in I_{e}$.

Lemma 9. Let $e$ be any idempotent in $F(X, Y)$ and $\alpha \epsilon$ $F(X, Y)$. Then condition (2) in Lemma 8 is equivalent to the statement

$$
(X \backslash Y) \alpha \subseteq X e, \quad \alpha \text { is injective on } Y \backslash(X e) \alpha^{-1} .
$$

Proof. Assume that, for $y \in Y$, if $y \alpha \notin X e$, then $\left|(y \alpha) \alpha^{-1}\right|=1$. We prove $(X \backslash Y) \alpha \subseteq X$ e. Let $x \alpha \in(X \backslash Y) \alpha$ for some $x \in X \backslash Y$. Since $(X \backslash Y) \alpha \subseteq X \alpha=Y \alpha$, there exists $y \in Y$ such that $x \alpha=y \alpha$. Thus $x, y \in(y \alpha) \alpha^{-1}$. If $y \alpha \notin X e$, then $\left|(y \alpha) \alpha^{-1}\right|=1$ by the assumption. Whence $x=y$, this is a contradiction. Hence $y \alpha \in X e$; that is, $x \alpha \in X e$. Next, we prove $\alpha$ is injective on $Y \backslash(X e) \alpha^{-1}$. Let $w, z \in Y \backslash(X e) \alpha^{-1}$ be such that $w \alpha=z \alpha$. Then $w \in Y$ and $w \alpha \notin X e$. Since $w, z \in(w \alpha) \alpha^{-1}$, we obtain $w=z$ by our assumption.

Conversely, suppose that $(X \backslash Y) \alpha \subseteq X e$ and $\alpha$ is injective on $Y \backslash(X e) \alpha^{-1}$. Let $y \in Y$ and $y \alpha \notin X e$. To show that $\left|(y \alpha) \alpha^{-1}\right|=1$, we let $u, v \in(y \alpha) \alpha^{-1}$. Then $u \alpha=v \alpha=y \alpha \notin$ $X e$; this implies that $u, v \notin(X e) \alpha^{-1}$. Since $(X \backslash Y) \alpha \subseteq X e$, we obtain $u, v \in Y$. Thus $u, v \in Y \backslash(X e) \alpha^{-1}$ and so $u=v$ since $\alpha$ is injective on $Y \backslash(X e) \alpha^{-1}$.

Lemma 10. Let $\alpha$ be any element in $T(X)$ and let $e$ be an idempotent in $T(X)$. If there is $\alpha^{\prime} \in V(\alpha)$ such that $e=e \alpha^{\prime} \alpha=$ $\alpha \alpha^{\prime} e, e \alpha=\alpha e$ and $e \alpha^{\prime}=\alpha^{\prime} e$, then $\left.\alpha\right|_{X e} \in G(X e)$.

Proof. Assume that the conditions hold. Since

$$
\begin{aligned}
X e & =X\left(e \alpha^{\prime} \alpha\right)=X\left(e \alpha^{\prime}\right) \alpha=X\left(\alpha^{\prime} e\right) \alpha \subseteq(X e) \alpha \\
& =X(e \alpha)=X(\alpha e) \subseteq X e,
\end{aligned}
$$

it follows that $(\mathrm{Xe}) \alpha=X e$; that is, $\alpha$ maps $X e$ onto $X e$. We prove that $\left.\alpha\right|_{X e}$ is injective. Let $x, y \in X e$ be such that $x \alpha=$ $y \alpha$. Then

$$
\begin{aligned}
x & =x e=x\left(\alpha \alpha^{\prime} e\right)=(x \alpha) \alpha^{\prime} e=(y \alpha) \alpha^{\prime} e=y\left(\alpha \alpha^{\prime} e\right) \\
& =y e=y .
\end{aligned}
$$

Hence $\left.\alpha\right|_{X e}: X e \rightarrow X e$ is bijective.

Lemma 11. Let e be an idempotent in $F(X, Y)$. Then $F_{e}$ is an inverse subsemigroup of $F(X, Y)$ with minimum idempotent $e$.

Proof. Let $\alpha$ be any element in $F_{e}$. Then $\alpha \in I_{e}$. Since $I_{e}$ is an inverse semigroup, there exists $\beta \in I_{e}$ such that $\alpha=\alpha \beta \alpha$ 
and $\beta=\beta \alpha \beta$. We prove $\beta \in F(X, Y)$. Since $\beta=\beta \alpha \beta$, we obtain $X \beta=X \beta \alpha \beta \subseteq X \alpha \beta \subseteq Y \beta \subseteq X \beta$; that is, $X \beta=Y \beta$. To show $X \beta \subseteq Y$, we let $x \beta \in X \beta$ for some $x \in X$. Since $\alpha \in F_{e},(X \backslash Y) \alpha \subseteq X e$ by Lemma 9. If $x \beta \in X \backslash Y$, then $(x \beta) \alpha \in(X \backslash Y) \alpha \subseteq X e$. It follows that $x \beta=(x \beta \alpha) \beta \epsilon$ $(X e) \beta=X e \subseteq Y$; this is a contradiction. Hence, $x \beta \in Y$ and so $X \beta \subseteq Y$. Thus, $\beta \in I_{e} \cap F(X, Y)=F_{e}$. Whence $F_{e}$ is a regular subsemigroup of $I_{e}$, it follows from Lemma 7 that $F_{e}$ is an inverse subsemigroup of $F(X, Y)$ and $e$ is the minimum idempotent in $F_{e}$.

Lemma 12. Let $e$ be an idempotent in $F(X, Y)$. If $M$ is any inverse subsemigroup of $F(X, Y)$ such that $F_{e} \subseteq M$, then $E(M) \subseteq F_{e}$.

Proof. Assume that $M$ is an inverse subsemigroup of $F(X, Y)$ containing $F_{e}$. Let $f$ be any idempotent in $M$. Since $e, f$ are idempotents in $M$, we get $e f=f e$. We prove that $X e \subseteq X f$. Suppose that $X e \nsubseteq X f$. Then $X e f \subseteq X e \cap X f \subseteq X e$, so there exists $z \in X e \backslash X e f$. We see that $z f \in X e f$ and $z f \neq z$. Now, we have $z$, zef $\in$ Xe such that $z \neq z e f$. Let $\sigma \in G(X e)$ be such that $z \sigma=z e f$ and $(z e f) \sigma=z$. We define $\alpha \in T(X)$ by

$$
x \alpha=(x e) \sigma \quad \forall x \in X
$$

We see that $X \alpha=(X e) \sigma=(Y e) \sigma=Y \alpha$ and $X \alpha=(X e) \sigma=$ $X e \subseteq Y$; we obtain $\alpha \in F(X, Y)$. We prove $\left.\alpha\right|_{X e} \in G(X e)$. Let $x, y \in X$ be such that $(x e) \alpha=(y e) \alpha$. Then $((x e) e) \sigma=$ $((y e) e) \sigma$; that is, $(x e) \sigma=(y e) \sigma$. Thus $x e=y e$ since $\sigma$ is injective on $X e$. Since $(X e) \alpha=((X e) e) \sigma=(X e) \sigma=X e, \alpha$ is surjective on $X e$. Hence, $\left.\alpha\right|_{X e} \in G(X e)$. Since $y \alpha=(y e) \sigma \in$ $X e$ for all $y \in Y$, whence condition (2) in Lemma 8 holds. To prove $\alpha e=e \alpha$. Let $x \in X$. Since $(x e) \sigma \in X e$ and $e$ is an idempotent, we get that $x \alpha e=((x e) \sigma) e=(x e) \sigma=((x e) e) \sigma=$ $x e \alpha$. So, $\alpha \in F_{e}$. By Lemma 11, we have $F_{e}$ as an inverse subsemigroup of $F(X, Y)$. Then there exists $\alpha^{-1} \in V(\alpha) \cap F_{e}$ and $\sigma^{-1}=\left.\alpha^{-1}\right|_{X e} \in G(X e)$. Since $z e f=z f e \in X e$, we obtain $(z e f) \alpha^{-1}=(z e f) \sigma^{-1}=z$. It is clear that $\alpha^{-1} f \alpha$ is an idempotent in $M$. We see that

$$
\begin{aligned}
(z e f) f\left(\alpha^{-1} f \alpha\right) & =(z e f)\left(\alpha^{-1} f \alpha\right)=(z f) \alpha \\
& =[(z f) e] \sigma=(z e f) \sigma=z, \\
(z e f)\left(\alpha^{-1} f \alpha\right) f & =(z f) \alpha f=[(z f e) \sigma] f \\
& =[(z e f) \sigma] f=z f .
\end{aligned}
$$

Since $z \neq z f$, this implies that $f\left(\alpha^{-1} f \alpha\right) \neq\left(\alpha^{-1} f \alpha\right) f$ which is a contradiction. Therefore, $X e \subseteq X f$. Hence $e f=e$ and so $e=e f=f e$. It is easy to see that $\left.f\right|_{X e}=i d_{X e}$, the identity map on $X e$, which implies that $\left.f\right|_{X e} \in G(X e)$. Thus, conditions (1) and (3) in Lemma 8 are satisfied for $f$.

In order to verify condition (2) in Lemma 8 for $f$ to be an element of $F_{e}$, let $y \in Y$ be such that $y f \notin X e$. We show that $\left|(y f) f^{-1}\right|=1$. Let $u, v \in(y f) f^{-1}$. Then $u f=v f=y f \notin X e$.
Suppose that $u \neq v$. Assume that $v \in Y$. We define $d \in T(X)$ by

$$
x d= \begin{cases}x & \text { if } x \in Y \backslash\{u\}, \\ x e & \text { if } x \in\{u\} \cup(X \backslash Y) .\end{cases}
$$

Obviously, $X d \subseteq Y$ and it is easy to verify that $(x e) d=x e=$ $x(d e)$ for all $x \in X$. Hence, $(X e) d=X e$ and $e d=d e$. It is easy to show that $\left.d\right|_{X e}$ is injective, whence $\left.d\right|_{X e} \in G(X e)$. We prove $X d \subseteq Y d$. Let $x d \in X d$ for some $x \in X$. If $x \in Y \backslash\{u\}$, then $x d \in Y d$. But if $x \in\{u\} \cup(X \backslash Y)$, then $x d=x e=$ $(x e) d \in Y d$. Thus, $X d \subseteq Y d$ and so $d \in F(X, Y)$. We prove $d$ satisfies the condition in Lemma 9. By the definition of $d$, we have $(X \backslash Y) d \subseteq X e$. Let $a, b \in Y \backslash(X e) d^{-1}$. Then $a, b \in Y$ and $a d, b d \notin X e$, which implies that $a, b \in Y \backslash\{u\}$. So, if $a d=b d$, then $a=b$. Hence, $d$ is injective on $Y \backslash(X e) d^{-1}$ and $d \in F_{e}$. Next, we prove $d$ is an idempotent. For $x \in Y \backslash\{u\},(x d) d=$ $x d$. If $x \in\{u\} \cup(X \backslash Y)$, then $(x d) d=(x e) d=x e=x d$. Thus, $d^{2}=d$. Since $d$ and $f$ are idempotents in $M, d f=f d$. However,

$$
\begin{aligned}
& u d f=(u e) f=u e \in X e, \\
& u f d=(v f) d=v d f=v f \notin X e,
\end{aligned}
$$

a contradiction. Hence, $v \in X \backslash Y$. Define $g \in T(X)$ by

$$
x g= \begin{cases}x & \text { if } x \in Y, \\ x e & \text { if } x \in X \backslash Y .\end{cases}
$$

Similarly, by using the same argument as in the proof above for $d$, we can show that $g$ is an idempotent in $F_{e}$. Since $f, g \in$ $E(M)$, we obtain $f g=g f$. We see that

$$
\begin{aligned}
v f g & =v f \notin X e \\
\text { but } v g f & =(v e) f=v e \in X e,
\end{aligned}
$$

and this is a contradiction. Thus, $u=v$ and so condition (2) in Lemma 8 is also satisfied. Whence $f \in F_{e}$, therefore, $E(M) \subseteq$ $F_{e}$ as required.

Now, we are ready to prove our main result (Theorem 13).

Theorem 13. Let $e$ be an idempotent in $F(X, Y)$. Then $F_{e}$ is a maximal inverse subsemigroup of $F(X, Y)$ with minimum idempotente.

Proof. From Lemma 11, $F_{e}$ is an inverse subsemigroup of $F(X, Y)$ with minimum idempotent $e$. To prove the maximality, let $M$ be any inverse semigroup of $F(X, Y)$ such that $F_{e} \subseteq M \subsetneq F(X, Y)$. Then by Lemma 12, we have $E(M) \subseteq F_{e}$, which implies that $e$ is the minimum idempotent in $M$. We prove $M=F_{e}$ by letting $\alpha \in M$. Since $\alpha, \alpha^{-1} \in M$, we obtain $e \alpha=\alpha e$ and $e \alpha^{-1}=\alpha^{-1} e$ by Lemma 2. And since $\alpha \alpha^{-1}$ and $\alpha^{-1} \alpha$ are idempotents in $M, e=\alpha \alpha^{-1} e$ and $e=e \alpha^{-1} \alpha$. Then by Lemma 10, we get that $\left.\alpha\right|_{X e} \in G(X e)$. Thus, conditions (1) and (3) in Lemma 8 are satisfied for $\alpha$ to be an element of $F_{e}$. Next, we prove that $\alpha$ satisfies condition (2) in Lemma 8. 
Let $y \in Y$ be such that $y \alpha \notin X e$. Let $u, v \in(y \alpha) \alpha^{-1}$. Then $u \alpha=v \alpha=y \alpha \notin X e$; this implies that $u \alpha \alpha^{-1}=v \alpha \alpha^{-1}=$ $y \alpha \alpha^{-1} \notin X e$. Now, we have $\alpha \alpha^{-1} \in E\left(F_{e}\right), y \in Y$ and $y\left(\alpha \alpha^{-1}\right) \notin X e$. Since $u, v \in\left[y\left(\alpha \alpha^{-1}\right)\right]\left(\alpha \alpha^{-1}\right)^{-1}$, we obtain that $u=v$ by using condition (2) in Lemma 8 for $\alpha \alpha^{-1} \in F_{e}$. Hence, $\alpha \in F_{e}$ and so $M \subseteq F_{e}$. Therefore, $M=F_{e}$ and $F_{e}$ is a maximal inverse subsemigroup of $F(X, Y)$.

Corollary 14. Let e be an idempotent in $F(X, Y)$. Then $F_{e}$ is a maximal inverse subsemigroup of $T(X, Y)$.

Proof. It follows directly from Theorem 13 and Proposition 6.

Remark 15. $F_{e} \neq F_{f}$ for all distinct idempotents $e, f \in$ $F(X, Y)$. In fact, if there exist idempotents $e$ and $f$ in $F(X, Y)$ such that $F_{e}=F_{f}$, then $f \in F_{e}$ and $e \in F_{f}$ such that $e$ and $f$ are minimum idempotents of $F_{e}$ and $F_{f}$, respectively. Thus, we get $e \leq f$ and $f \leq e$; it follows that $e=f$.

Corollary 16. Let $e$ be an idempotent in $F(X, Y)$ such that $X e=Y$. Then $F_{e}=H_{e}$ is a maximal inverse subsemigroup of $F(X, Y)$.

Proof. Since $F_{e}$ is a maximal inverse subsemigroup of $F(X, Y)$ with the minimum idempotent $e$, we obtain that $H_{e} \subseteq F_{e}$ by Lemma 1. To show that $F_{e} \subseteq H_{e}$, let $\alpha \in F_{e}$. Then $\left.\alpha\right|_{X e} \in$ $G(X e)$ and $\alpha e=e \alpha$. Since $(X e) \alpha=X e$, it follows that $X \alpha=$ $Y \alpha=(X e) \alpha=X e$. We prove that $\pi_{\alpha}=\pi_{e}$. Let $x, y \in X$ such that $x \alpha=y \alpha$. Then $(x e) \alpha=(x \alpha) e=(y \alpha) e=(y e) \alpha$; this implies that $x e=y e$ since $\left.\alpha\right|_{X e}$ is injective. Hence $\pi_{\alpha} \subseteq$ $\pi_{e}$. On the other hand, let $x e=y e$. Then $(x \alpha) e=(x e) \alpha=$ $(y e) \alpha=(y \alpha) e$. Since $x \alpha, y \alpha \in Y=X e$ and $e$ is an idempotent, we obtain $x \alpha=y \alpha$. Hence $\pi_{e} \subseteq \pi_{\alpha}$ and $\pi_{\alpha}=\pi_{e}$. Then by Lemma 4, we get that $\alpha \in H_{e}$. Thus, $F_{e} \subseteq H_{e}$. Therefore, $H_{e}=$ $F_{e}$ is the maximal inverse subsemigroup of $F(X, Y)$.

Notice that if $e$ is a constant map with range $\{a\} \subseteq Y$, then $I_{e}=I_{a}$ and

$$
\begin{aligned}
F_{e} & =F(X, Y) \cap I_{a}=\{\alpha \in F(X, Y): a \alpha \\
& \left.=a, \alpha \text { is injective on } X \backslash a \alpha^{-1}\right\} ;
\end{aligned}
$$

that is, $F_{e}=F_{a}$. Thus, we get the following corollary which appeared in [14, Theorem 4.3].

Corollary 17. If $e \in F(X, Y)$ is a constant map with range $\{a\} \subseteq Y$, then $F_{e}=F_{a}$ is a maximal inverse subsemigroup of $F(X, Y)$ and $T(X, Y)$.

Next, we count the number of elements in $F_{e}$. Let $e$ be any idempotent in $F(X, Y)$. We know that $H_{e} \cong G(X e)$; see [17, Section 4]. Thus, if $X e=Y$, then $F_{e}=H_{e} \cong G(X e)=G(Y)$ by Corollary 16. Hence, $\left|F_{e}\right|=|G(Y)|$ when $X e=Y$. In what follows, we assume that $\mathrm{Xe} \subsetneq Y$.

Recall that the number of ways that $r$ objects can be chosen from $n$ distinct objects written as $\left(\begin{array}{c}n \\ r\end{array}\right)$ is given by

$$
\left(\begin{array}{l}
n \\
r
\end{array}\right)=\frac{n !}{(n-r) ! r !} \text {. }
$$

Let $A$ and $B$ be (possibly empty) sets and let $\operatorname{PI}(A, B)$ be the set of all partial injective maps from $A$ into $B$. We note that $\operatorname{PI}(A, B)$ contains the empty map. Moreover, if $A=\emptyset$ or $B=\emptyset$, then $|\operatorname{PI}(A, B)|=1$. And if $A$ and $B$ are finite sets such that $|A|=m$ and $|B|=n$, then we shall write $\operatorname{PI}(m, n)$ instead of $\operatorname{PI}(A, B)$. By a consideration of the cardinality of a domain of partial injective maps, we can verify that

$$
|\mathrm{PI}(m, n)|=\sum_{j=0}^{\min \{m, n\}}\left(\begin{array}{l}
m \\
j
\end{array}\right)\left(\begin{array}{l}
n \\
j
\end{array}\right) j ! \text { for } m, n \geq 1 .
$$

Since $X e \subsetneq Y$, there exists $x \in Y \backslash X e$ such that $x e \in X e$. We see that $x \in\left((x e) e^{-1} \backslash\{x e\}\right) \cap Y$. For each $y \in X e$, we define

$$
\begin{aligned}
& A(y)=\left(y e^{-1} \backslash\{y\}\right) \cap Y, \\
& B(y)=y e^{-1} \cap(X \backslash Y) .
\end{aligned}
$$

Then $y e^{-1}=\{y\} \cup A(y) \cup B(y)$ is a disjoint union such that $y e^{-1} \cap X e=\{y\}$ and $A(y) \cap X e=\emptyset=B(y) \cap X e$. Moreover, $A(y) \neq \emptyset$ for some $y \in X e$ since $X e \varsubsetneqq Y$. Let

$$
\begin{aligned}
& \mathscr{A}=\left\{y_{i} \in X e: A\left(y_{i}\right) \neq \emptyset, i \in I\right\}, \\
& \mathscr{B}=\left\{y_{j} \in X e: B\left(y_{j}\right) \neq \emptyset, j \in J\right\} .
\end{aligned}
$$

Then $y_{i} e=y_{i}$ and $y_{i} e^{-1} \cap X e=\left\{y_{i}\right\}$ for all $i \in I$. It is easy to see that

$$
\begin{aligned}
X \backslash X e= & \left(\bigcup_{i \in I} A\left(y_{i}\right)\right) \\
& \cup\left(\bigcup_{j \in J} B\left(y_{j}\right)\right) \text { is adisjoint union. }
\end{aligned}
$$

Let $\alpha$ be any element in $F_{e}$. Then $\alpha e=e \alpha$ and $\left.\alpha\right|_{X e}=\sigma$ for some $\sigma \in G(X e)$. For each $x \in X \backslash X e, x \in A\left(y_{i}\right) \cup B\left(y_{j}\right)$ for some $y_{i} \in \mathscr{A}$ and $y_{j} \in \mathscr{B}$. If $x \in A\left(y_{i}\right)$, then $x e=y_{i} \in X e$. Since $\alpha e=e \alpha$, we obtain $(x \alpha) e=(x e) \alpha=y_{i} \alpha=y_{i} \sigma$; that is, $x \alpha \in\left(y_{i} \sigma\right) e^{-1}=\left\{y_{i} \sigma\right\} \cup A\left(y_{i} \sigma\right) \cup B\left(y_{i} \sigma\right)$. Since $x \alpha \in Y$ and $B\left(y_{i} \sigma\right) \subseteq X \backslash Y$, we obtain that $x \alpha \in\left\{y_{i} \sigma\right\} \cup A\left(y_{i} \sigma\right)$. If $x \in B\left(y_{j}\right) \subseteq X \backslash Y$, then $x \alpha \in(X \backslash Y) \alpha \subseteq X e$ by Lemma 9 . Since $e$ is an idempotent, we get $x \alpha=(x \alpha) e=(x e) \alpha=y_{j} \alpha=$ $y_{j} \sigma$. By condition (2) in Lemma 8 , we see that $\alpha$ is injective on all elements of $A\left(y_{i}\right)$ which are not mapped into $\left\{y_{i} \sigma\right\}$ for all $i \in I$. Thus for each $i \in I,\left.\alpha\right|_{A\left(y_{i}\right)}$ corresponds with an element $\theta_{i}$ of $\operatorname{PI}\left(A\left(y_{i}\right), A\left(y_{i} \sigma\right)\right)$; that is, for $u \in A\left(y_{i}\right)$,

$$
u \alpha= \begin{cases}u \theta_{i} & \text { if } u \in \operatorname{dom}\left(\theta_{i}\right) \\ y_{i} \sigma & \text { if } u \in A\left(y_{i}\right) \backslash \operatorname{dom}\left(\theta_{i}\right)\end{cases}
$$

We have shown the following. Given $\alpha \in F_{e}$. Then $\alpha$ is a union of a permutation $\sigma \in G(X e)$, a union of partial injections $\theta_{i}: A\left(y_{i}\right) \rightarrow A\left(y_{i} \sigma\right)$ for each $y_{i} \in \mathscr{A}$, and a union of functions from $B\left(y_{j}\right)$ into $\left\{y_{j} \sigma\right\}$ for each $y_{j} \in \mathscr{B}$.

Theorem 18. Lete be any idempotent in $F(X, Y)$ with $X e \subsetneq Y$. Let $G(X e)=\left\{\sigma_{k}: k \in \Lambda\right\}$ and $\mathscr{A}=\left\{y_{i} \in X e: A\left(y_{i}\right) \neq \emptyset, i \in\right.$ $I\}$. Then there is a one-to-one correspondence between $F_{e}$ and $\bigcup_{k \in \Lambda}\left[\left\{\sigma_{k}\right\} \times \prod_{i \in I} P I\left(A\left(y_{i}\right), A\left(y_{i} \sigma_{k}\right)\right)\right]$. 
Proof. For each $\alpha \in F_{e}$, we have $\left.\alpha\right|_{X e}=\sigma_{k}$ for some $k \in \Lambda$ and partial injections $\theta_{i}: A\left(y_{i}\right) \rightarrow A\left(y_{i} \sigma_{k}\right)$ for all $y_{i} \in \mathscr{A}$. We define

$$
\Psi: F_{e} \longrightarrow \bigcup_{k \in \Lambda}\left[\left\{\sigma_{k}\right\} \times \prod_{i \in I} \operatorname{PI}\left(A\left(y_{i}\right), A\left(y_{i} \sigma_{k}\right)\right)\right],
$$

by $\alpha \Psi=\left(\sigma_{k},\left(\theta_{i}\right)_{i \in I}\right)$ for all $\alpha \in F_{e}$. We verify that $\Psi$ is injective. Let $\alpha, \beta \in F_{e}$ be such that $\alpha \Psi=\beta \Psi$. Then $\left(\sigma_{k},\left(\theta_{i}\right)_{i \in I}\right)=\left(\sigma_{l},\left(\tau_{i}\right)_{i \in I}\right)$ for some $\sigma_{k}, \sigma_{l} \in G(X e),\left(\theta_{i}\right)_{i \in I} \in$ $\prod_{i \in I} \mathrm{PI}\left(A\left(y_{i}\right), A\left(y_{i} \sigma_{k}\right)\right)$ and $\left(\tau_{i}\right)_{i \in I} \in \prod_{i \in I} \mathrm{PI}\left(A\left(y_{i}\right), A\left(y_{i} \sigma_{l}\right)\right)$. Thus, $\sigma_{k}=\sigma_{l}$ and $\theta_{i}=\tau_{i}$ for all $i \in I$; it follows that $x \alpha=x \beta$ for all $x \in X e \cup\left(\bigcup_{i \in I} A\left(y_{i}\right)\right)=Y$. For $x \in X \backslash Y$, $x \in B\left(y_{j}\right)$ for some $y_{j} \in \mathscr{B}=\left\{y_{j} \in X e: B\left(y_{j}\right) \neq\right.$ $\emptyset, j \in J\}$. We get that $x \alpha=y_{j} \sigma_{k}=y_{j} \sigma_{l}=x \beta$. Whence $\alpha=\beta$, next, we prove $\Psi$ is surjective. Let $\sigma_{k} \in G(X e)$ and $\left(\theta_{i}\right)_{i \in I} \in \prod_{i \in I} \operatorname{PI}\left(A\left(y_{i}\right), A\left(y_{i} \sigma_{k}\right)\right)$. We define $\alpha \in T(X, Y)$ which is determined by $\sigma_{k}$ and $\theta_{i}$ for all $i \in I$ as follows. For $x \in X$,

$$
x \alpha= \begin{cases}x \sigma_{k} & \text { if } x \in X e \\ x \theta_{i} & \text { if } x \in \operatorname{dom}\left(\theta_{i}\right) \neq \emptyset \\ y_{i} \sigma_{k} & \text { if } x \in A\left(y_{i}\right),\left(x \notin \operatorname{dom}\left(\theta_{i}\right) \text { or } \theta_{i} \text { is the empty map }\right) \\ y_{j} \sigma_{k} & \text { if } x \in B\left(y_{j}\right) .\end{cases}
$$

We see that, for each $x \in X \backslash Y, x \in B\left(y_{j}\right)$ for some $y_{j} \in \mathscr{B}$. Then $y_{j} \in X e \subseteq Y$ and $x \alpha=y_{j} \sigma_{k}=y_{j} \alpha$. So, $X \alpha \subseteq Y \alpha$, that is $\alpha \in F(X, Y)$. By the construction of $\alpha$, conditions (1) and (2) in Lemma 8 are satisfied for $\alpha$ to be an element of $F_{e}$. To prove $\alpha e=e \alpha$, we let $x \in X$. If $x \in X e$, then $(x \alpha) e=$ $\left(x \sigma_{k}\right) e=x \sigma_{k}=x \alpha=(x e) \alpha$, since $x, x \sigma_{k} \in X e$ and $e$ is an idempotent. If $x \in \operatorname{dom}\left(\theta_{i}\right) \neq \emptyset$, then $x \in A\left(y_{i}\right)$; it follows that $x e=y_{i}$ and $x \theta_{i} \in A\left(y_{i}\right) \theta_{i} \subseteq A\left(y_{i} \sigma_{k}\right)$. Thus, $\left(x \theta_{i}\right) e=y_{i} \sigma_{k}$ and $(x \alpha) e=\left(x \theta_{i}\right) e=y_{i} \sigma_{k}=y_{i} \alpha=(x e) \alpha$ since $y_{i} \in X e$. If $x \in A\left(y_{i}\right)$ and ( $x \notin \operatorname{dom}\left(\theta_{i}\right)$ or $\theta_{i}$ is the empty map), then $x e=y_{i}$ and $(x \alpha) e=\left(y_{i} \sigma_{k}\right) e=y_{i} \sigma_{k}=y_{i} \alpha=(x e) \alpha$. And if $x \in B\left(y_{j}\right)$, then $x e=y_{j}$ and $(x \alpha) e=\left(y_{j} \sigma_{k}\right) e=y_{j} \sigma_{k}=y_{j} \alpha=$ $(x e) \alpha$, since $y_{j} \in X e$. Hence, $\alpha e=e \alpha$. Whence $\alpha \in F_{e}$ and $\alpha \Psi=\left(\sigma_{k},\left(\theta_{i}\right)_{i \in I}\right)$, therefore, $\Psi$ is a bijection.

The following corollary is a straightforward consequence of Theorem 18.

Corollary 19. Let $e$ be an idempotent in $F(X, Y)$ such that $X e \subsetneq Y$ and $|X e|=p \in \mathbb{Z}^{+}$. Let $\mathscr{A}=\left\{y_{i} \in X e: A\left(y_{i}\right) \neq\right.$ $\emptyset, 1 \leq i \leq t \leq p\}$. For each $y_{i} \in \mathscr{A}$, we let $\left|A\left(y_{i}\right)\right|=m_{i} \geq 1$ and $\left|A\left(y_{i} \sigma_{k}\right)\right|=n_{i, k} \geq 0(1 \leq i \leq t, 1 \leq k \leq p$ !). Then

$$
\left|F_{e}\right|=\sum_{k=1}^{p !}\left(\prod_{i=1}^{t}\left|P I\left(m_{i}, n_{i, k}\right)\right|\right) .
$$

It is known that if $X=Y$, then $F(X, Y)=T(X)$ and $F_{e}=$ $I_{e}$. Then by Corollary 19 , we have the following corollary.

Corollary 20. Lete be an idempotent in $T(X)$ such that Xe $\subsetneq$ $X$ and $|X e|=p \in \mathbb{Z}^{+}$. Let $\mathscr{A}=\left\{y_{i} \in X e: A\left(y_{i}\right) \neq \emptyset, 1 \leq\right.$ $i \leq t \leq p\}$. For each $y_{i} \in \mathscr{A}$, we let $\left|A\left(y_{i}\right)\right|=m_{i} \geq 1$ and $\left|A\left(y_{i} \sigma_{k}\right)\right|=n_{i, k} \geq 0(1 \leq i \leq t, 1 \leq k \leq p !)$. Then

$$
\left|I_{e}\right|=\sum_{k=1}^{p !}\left(\prod_{i=1}^{t}\left|P I\left(m_{i}, n_{i, k}\right)\right|\right) \text {. }
$$

If $e$ is a constant map with range $\{a\} \subseteq Y$, then we get the following corollary which appeared in [14, Theorem 4.5].
Corollary 21. Let $Y$ be a finite subset of $X$ with $|Y|=r \geq 2$ and $e$ a constant map in $F(X, Y)$ with range $\{a\} \subseteq Y$; then $\left|F_{a}\right|=\sum_{j=0}^{r-1} j !\left(\begin{array}{c}r-1 \\ j\end{array}\right)^{2}$.

Proof. We note that $F_{e}=F_{a}$ by Corollary 17. Since $X e=\{a\}$, we obtain $|X e|=1$ and there is a unique $A(a)=Y \backslash\{a\}$; that is, $|A(a)|=r-1$. By Corollary 19, we have

$$
\begin{aligned}
\left|F_{a}\right| & =\left|F_{e}\right|=\sum_{k=1}^{1 !}\left(\prod_{i=1}^{1}|\operatorname{PI}(A(a), A(a))|\right) \\
& =|\operatorname{PI}(A(a), A(a))|=|\mathrm{PI}(r-1, r-1)|,
\end{aligned}
$$

where

$$
\begin{aligned}
|\mathrm{PI}(r-1, r-1)| & =\sum_{j=0}^{\min \{r-1, r-1\}}\left(\begin{array}{c}
r-1 \\
j
\end{array}\right)\left(\begin{array}{c}
r-1 \\
j
\end{array}\right) j ! \\
& =\sum_{j=0}^{r-1}\left(\begin{array}{c}
r-1 \\
j
\end{array}\right)^{2} j ! .
\end{aligned}
$$

Therefore, $\left|F_{a}\right|=\sum_{j=0}^{r-1} j !\left(\begin{array}{c}r-1 \\ j\end{array}\right)^{2}$.

\section{Maximal Regular of Ideals}

Throughout this section, $X$ is a finite set with $n$ elements and $Y$ a nonempty subset of $X$ with $r \geq 2$ elements. For convenience, we write $F_{n, r}$ for $F(X, Y)$.

For each $\alpha \in F_{n, r}$ with $|X \alpha|=k \in \mathbb{Z}^{+}$, it follows from Lemma 3 that we can write

$$
\alpha=\left(\begin{array}{cccc}
A_{1} & A_{2} & \cdots & A_{k} \\
a_{1} & a_{2} & \cdots & a_{k}
\end{array}\right)
$$

where $a_{1}, a_{2}, \ldots, a_{k} \in Y$ and $\left\{A_{1}, A_{2}, \ldots, A_{k}\right\}$ is a partition of $X$ such that $A_{i} \cap Y \neq \emptyset$ for all $1 \leq i \leq k$. 
For $1 \leq k \leq r$, we define $J(F ; k)=\left\{\alpha \in F_{n, r}:|X \alpha|=k\right\}$. Then $J(F ; k)$ is a $\mathscr{J}$-class of the semigroup $F_{n, r}$. Let $Q(F ; k)=$ $J(F ; 1) \cup J(F ; 2) \cup \cdots \cup J(F ; k)=\left\{\alpha \in F_{n, r}:|X \alpha| \leq k\right\}$, where $1 \leq k \leq r$. Then $Q(F ; k)$ is an ideal of $F_{n, r}$ and it is a regular subsemigroup of $F_{n, r}$; see [17, Lemma 7].

Recall that the principal factor of $F_{n, r}$ is the Rees quotient

$$
P_{k}=Q(F ; k) / Q(F ; k-1) \text {, }
$$

where $2 \leq k \leq r$. It is usually convenient to think of it as $J(F ; k) \cup\{0\}$, and the product of two elements of $P_{k}$ is taken to be zero if it falls in $Q(F ; k-1)$. Since $P_{k}$ is finite and it is not a zero semigroup, we obtain that $P_{k}$ is a completely 0 -simple semigroup; see [18, p. 233] for more details.

We need the following lemmas for proof of our main result.

Lemma 22 (see [1, p. 98]). Let $S$ be a completely 0-simple semigroup. If $a, b \in S$ and $a b \neq 0$, then $a b \in R_{a} \cap L_{b}$.

Lemma 23 (see [1, Proposition 2.3.7]). Let $a, b$ be elements in D-class $D$. Then $a b \in R_{a} \cap L_{b}$ if and only if $L_{a} \cap R_{b}$ contains an idempotent.

Lemma 24. Let $a$ be any element in a semigroup $S$ and $T a$ regular subsemigroup of $S$.

(1) If $L_{a} \cap T \neq \emptyset$, then $L_{a} \cap T$ contains an idempotent.

(2) If $R_{a} \cap T \neq \emptyset$, then $R_{a} \cap T$ contains an idempotent.

Proof. (1) Assume that $L_{a} \cap T \neq \emptyset$. Then there exists $b \in L_{a} \cap$ $T$. So, $b \mathscr{L} a$ and $b=b b^{\prime} b$ for some $b^{\prime} \in T$. We get that $b^{\prime} b$ is an idempotent such that $b^{\prime} b \in T$ and $b^{\prime} b \mathscr{L} b \mathscr{L} a$. Therefore, $b^{\prime} b$ is an idempotent in $L_{a} \cap T$.

(2) If there is $b \in R_{a} \cap T$, then we can write $b=b b^{\prime} b$ for some $b^{\prime} \in T$ and show that $b b^{\prime}$ is an idempotent in $R_{a} \cap$ $T$.

Lemma 25 (see [18, Lemma 3.5]). For $1 \leq k \leq r-1, Q(F ; k)=$ $\langle J(F ; k)\rangle$.

Lemma 26 (see [18, Lemma 4.2]). If $\alpha \in J(F ; k)$, then $\alpha$ can be written as a finite product of idempotents in $J(F ; k)$.

The following fact can be obtained from Lemmas 25 and 26 immediately.

Lemma 27. For $1 \leq k \leq r-1, Q(F ; k)=\langle E(J(F ; k))\rangle$.

Lemma 28. Let $1 \leq k \leq r-1$ and $\alpha \in J(F ; k)$. If $\beta \in L_{\alpha}$, then $\beta=\gamma \alpha$ for some $\gamma \in J(F ; k)$ such that $L_{\gamma} \neq L_{\alpha}$.

Proof. Suppose that $\beta \in L_{\alpha}$. Thus $X \alpha=X \beta$; we write

$$
\begin{aligned}
\alpha & =\left(\begin{array}{llll}
A_{1} & A_{2} & \cdots & A_{k} \\
a_{1} & a_{2} & \cdots & a_{k}
\end{array}\right), \\
\beta & =\left(\begin{array}{llll}
B_{1} & B_{2} & \cdots & B_{k} \\
a_{1} & a_{2} & \cdots & a_{k}
\end{array}\right),
\end{aligned}
$$

where $a_{1}, a_{2}, \ldots, a_{k} \in Y$ and $A_{i} \cap Y \neq \emptyset \neq B_{i} \cap Y$ for all $1 \leq i \leq k$. Since $X \alpha \neq Y$, there is $y \in Y \backslash X \alpha$ such that $y \in A_{j}$ for some $j \in\{1,2, \ldots, k\}$. We choose $d_{a_{i}} \in A_{i} \cap Y$ for all $i \in\{1,2, \ldots, j-1, j+1, \ldots, k\}$ and define

$$
\gamma=\left(\begin{array}{ccccccc}
B_{1} & \cdots & B_{j-1} & B_{j} & B_{j+1} & \cdots & B_{k} \\
d_{a_{1}} & \cdots & d_{a_{j-1}} & y & d_{a_{j+1}} & \cdots & d_{a_{k}}
\end{array}\right) .
$$

Then $\gamma \in J(F ; k)$ such that $X \gamma \neq X \alpha$ and $\beta=\gamma \alpha$.

Lemma 29. Let $2 \leq k \leq r-1$ and $\alpha \in J(F ; k)$. If $\beta \in R_{\alpha}$, then $\beta=\alpha \gamma$ for some $\gamma \in J(F ; k)$ such that $R_{\gamma} \neq R_{\alpha}$.

Proof. Assume that $\beta \in R_{\alpha}$. Thus $\pi_{\alpha}=\pi_{\beta}$; we write

$$
\begin{aligned}
& \alpha=\left(\begin{array}{llll}
A_{1} & A_{2} & \cdots & A_{k} \\
a_{1} & a_{2} & \cdots & a_{k}
\end{array}\right), \\
& \beta=\left(\begin{array}{llll}
A_{1} & A_{2} & \cdots & A_{k} \\
b_{1} & b_{2} & \cdots & b_{k}
\end{array}\right),
\end{aligned}
$$

where $a_{i}, b_{i} \in Y$ and $A_{i} \cap Y \neq \emptyset$ for all $1 \leq i \leq k$. Define

$$
\gamma=\left(\begin{array}{ccccc}
a_{1} & a_{2} & \cdots & a_{k-1} & \left\{a_{k}\right\} \cup(X \backslash X \alpha) \\
b_{1} & b_{2} & \cdots & b_{k-1} & b_{k}
\end{array}\right) .
$$

It is clear that $\gamma \in J(F ; k)$. If $\gamma \notin R_{\alpha}$, then $R_{\gamma} \neq R_{\alpha}$ and $\beta=\alpha \gamma$. If such $\gamma \in R_{\alpha}$, we define

$$
\begin{aligned}
& \gamma^{\prime} \\
& =\left(\begin{array}{ccccc}
\left\{a_{1}, y\right\} & a_{2} & \cdots & a_{k-1} & \left\{a_{k}\right\} \cup((X \backslash X \alpha) \backslash\{y\}) \\
b_{1} & b_{2} & \cdots & b_{k-1} & b_{k}
\end{array}\right),
\end{aligned}
$$

where $y \in Y \backslash X \alpha$. So, $\gamma^{\prime} \in J(F ; k)$ such that $R_{\gamma^{\prime}} \neq R_{\gamma}=R_{\alpha}$ and $\beta=\alpha \gamma^{\prime}$.

For the case $k=1$, the ideal $Q(F ; 1)=J(F ; 1)$ consisting of all constant maps $X_{a}$ with range $\{a\} \subseteq Y$. It is easy to verify that $J(F ; 1) \backslash\left\{X_{a}\right\}$ is a maximal regular subsemigroup of $Q(F ; 1)$ for all $a \in Y$.

Lemma 30. For $2 \leq k \leq r-1, M=Q(F ; k-1) \cup(J(F ; k) \backslash$ $\left.L_{\alpha}\right)$ is a maximal regular subsemigroup of $Q(F ; k)$ for all $\alpha \in$ $J(F ; k)$.

Proof. Let $\alpha$ be any element in $J(F ; k)$. Let $\beta, \gamma \in J(F ; k) \backslash L_{\alpha}$. If $\beta \gamma \in Q(F ; k-1)$, then $\beta \gamma \in M$. If $\beta \gamma \notin Q(F ; k-1)$, then $\beta \gamma \neq 0$ in $P_{k}=Q(F ; k) / Q(F ; k-1)$; that is, $\beta \gamma \in R_{\beta} \cap L_{\gamma}$ by Lemma 22. Thus, $\beta \gamma \in L_{\gamma} \neq L_{\alpha}$ and so $\beta \gamma \in M$. Hence, $M$ is a subsemigroup of $Q(F ; k)$. We prove $M$ is regular. Let $\lambda \in M$. If $\lambda \in Q(F ; k-1)$, then $\lambda$ is a regular element in $Q(F ; k-1)$. If $\lambda \in J(F ; k) \backslash L_{\alpha}$, we write

$$
\lambda=\left(\begin{array}{cccc}
A_{1} & A_{2} & \cdots & A_{k} \\
a_{1} & a_{2} & \cdots & a_{k}
\end{array}\right),
$$

where $a_{1}, a_{2}, \ldots, a_{k} \in Y$ and $A_{i} \cap Y \neq \emptyset$ for all $1 \leq i \leq k$. Since $X \alpha \neq Y$, there exists $y \in Y \backslash X \alpha$ such that $y \in A_{j}$ 
for some $j \in\{1,2, \ldots, k\}$. We choose $d_{a_{i}} \in A_{i} \cap Y$ for all $i \in\{1,2, \ldots, j-1, j+1, \ldots, k\}$ and define

$$
\begin{aligned}
& \mu \\
& =\left(\begin{array}{cccccccc}
a_{1} & \cdots & a_{j-1} & a_{j} & a_{j+1} & \cdots & a_{k-1} & \left\{a_{k}\right\} \cup(X \backslash X \lambda) \\
d_{a_{1}} & \cdots & d_{a_{j-1}} & y & d_{a_{j+1}} & \cdots & d_{a_{k-1}} & d_{a_{k}}
\end{array}\right) .
\end{aligned}
$$

Then $\mu \in J(F ; k), X \mu \neq X \alpha$, and $\lambda=\lambda \mu \lambda$. Hence, $M$ is a regular semigroup. Next, we prove $M$ is maximal. Let $S$ be a regular subsemigroup of $Q(F ; k)$ such that $M \subsetneq S \subseteq Q(F ; k)$. Then there exists $\xi \in S \backslash M$; that is, $\xi \in L_{\alpha} \cap S$. Let $\tau$ be any element in $L_{\alpha}=L_{\xi}$. Then there is $\gamma \in J(F ; k)$ such that $\gamma \notin L_{\xi}$ and $\tau=\gamma \xi$ by Lemma 28; that is, $\gamma \in M \subseteq S$. Thus, $\tau=\gamma \xi \in S$. Hence, $L_{\alpha} \subseteq S$ and therefore $S=Q(F ; k)$.

Lemma 31. For $2 \leq k \leq r-1, M=Q(F ; k-1) \cup\left(J(F ; k) \backslash R_{\alpha}\right)$ is a maximal regular subsemigroup of $Q(F ; k)$ for all $\alpha \in J(F ; k)$.

Proof. Let $\alpha$ be any element in $J(F ; k)$. Let $\beta, \gamma \in J(F ; k) \backslash R_{\alpha}$. If $\beta \gamma \in Q(F ; k-1)$, then $\beta \gamma \in M$. If $\beta \gamma \notin Q(F ; k-1)$, then $\beta \gamma \neq 0$ in $P_{k}$ and so $\beta \gamma \in R_{\beta} \cap L_{\gamma}$ by Lemma 22. Thus, $\beta \gamma \in R_{\beta} \neq R_{\alpha}$. So, $\beta \gamma \in M$. Hence, $M$ is a subsemigroup of $Q(F ; k)$. We prove $M$ is regular. Let $\lambda \in M$. If $\lambda \in Q(F ; k-1)$, then $\lambda$ is a regular element in $Q(F ; k-1)$. If $\lambda \in J(F ; k) \backslash R_{\alpha}$, we write

$$
\lambda=\left(\begin{array}{cccc}
A_{1} & A_{2} & \cdots & A_{k} \\
a_{1} & a_{2} & \cdots & a_{k}
\end{array}\right),
$$

where $a_{1}, a_{2}, \ldots, a_{k} \in Y$ and $A_{i} \cap Y \neq \emptyset$ for all $1 \leq i \leq k$. We choose $d_{a_{i}} \in A_{i} \cap Y$ for all $1 \leq i \leq k$ and define

$$
\lambda^{\prime}=\left(\begin{array}{ccccc}
a_{1} & a_{2} & \cdots & a_{k-1} & \left\{a_{k}\right\} \cup(X \backslash X \lambda) \\
d_{a_{1}} & d_{a_{2}} & \cdots & d_{a_{k-1}} & d_{a_{k}}
\end{array}\right) .
$$

Then $\lambda^{\prime} \in J(F ; k)$ and $\lambda=\lambda \lambda^{\prime} \lambda$. If $\lambda^{\prime} \notin R_{\alpha}$, then $\lambda^{\prime}$ is a regular element in $M$. If $\lambda^{\prime} \in R_{\alpha}$, we define

$$
\begin{aligned}
& \lambda^{\prime \prime} \\
& =\left(\begin{array}{ccccc}
\left\{a_{1}, y\right\} & a_{2} & \cdots & a_{k-1} & \left\{a_{k}\right\} \cup((X \backslash X \alpha) \backslash\{y\}) \\
d_{a_{1}} & d_{a_{2}} & \cdots & d_{a_{k-1}} & d_{a_{k}}
\end{array}\right),
\end{aligned}
$$

where $y \in Y \backslash X \lambda$ since $k<r$. We obtain $\lambda^{\prime \prime} \notin R_{\lambda^{\prime}}=R_{\alpha}$ and $\lambda=\lambda \lambda^{\prime \prime} \lambda$. Hence, $M$ is a regular semigroup. For a maximality of $M$, we let $S$ be a regular subsemigroup of $Q(F ; k)$ such that $M \subsetneq S \subseteq Q(F ; k)$. Then there exists $\xi \in S \backslash M$; that is, $\xi \in$ $R_{\alpha} \cap S$. Let $\tau$ be any element in $R_{\alpha}=R_{\xi}$. Then there is $\gamma \in$ $J(F ; k)$ such that $\gamma \notin R_{\xi}=R_{\alpha}$ and $\tau=\xi \gamma$ by Lemma 29; that is, $\gamma \in M \subseteq S$. Thus, $\tau \in S$ and so $R_{\alpha} \subseteq S$. Therefore, $S=Q(F ; k)$.

Theorem 32. Each maximal regular subsemigroup of $Q(F ; k)$, $2 \leq k \leq r-1$ must be one of the following forms:

$$
\begin{aligned}
& \text { (1) } Q(F ; k-1) \cup\left(J(F ; k) \backslash L_{\alpha}\right) \text {; } \\
& \text { (2) } Q(F ; k-1) \cup\left(J(F ; k) \backslash R_{\beta}\right) \text {, }
\end{aligned}
$$

where $\alpha, \beta \in J(F ; k), L_{\alpha}$ is the $\mathscr{L}$-class containing $\alpha$ in $J(F ; k)$, and $R_{\beta}$ is the $\mathscr{R}$-class containing $\beta$ in $J(F ; k)$.
Proof. By Lemmas 30 and 31, both (1) and (2) are maximal regular subsemigroups of $Q(F ; k)$.

On the other hand, let $M$ be an arbitrary maximal regular subsemigroup of $Q(F ; k)$. It is easy to see that $M \cup Q(F ; k-1)$ is a regular subsemigroup of $Q(F ; k)$ such that $M \subseteq M \cup$ $Q(F ; k-1) \subseteq Q(F ; k)$. Then $M=M \cup Q(F ; k-1)$ or $M \cup Q(F ; k-1)=Q(F ; k)$ by the maximality of $M$. If $M \cup Q(F ; k-1)=Q(F ; k)$, then $J(F ; k)=M \cap J(F ; k) \subseteq M$. We obtain $M \subseteq Q(F ; k)=\langle J(F ; k)\rangle \subseteq M$ by Lemma 25; this implies that $M=Q(F ; k)$, a contradiction. Whence $M=M \cup Q(F ; k-1)$ and so $Q(F ; k-1) \subseteq M$, assume that $L_{\alpha} \cap M \neq \emptyset$ and $R_{\alpha} \cap M \neq \emptyset$ for all $\alpha \in J(F ; k)$. We first prove that $E(J(F ; k)) \subseteq M$. Let $e$ be an idempotent in $J(F ; k)$. Thus, $L_{e} \cap M \neq \emptyset \neq R_{e} \cap M$. By Lemma $24, L_{e} \cap M$ and $R_{e} \cap M$ contain idempotents, say $f$ and $g$ respectively. So, $f \mathscr{L} e$ and gRe. Since $L_{f} \cap R_{g}=L_{e} \cap R_{e}$ contains the idempotent $e, f g \in R_{f} \cap L_{g} \subseteq J(F ; k)$ by Lemma 23. Since $f g \in M$ and $M$ is regular, $f g=(f g) h(f g)$ for some $h \in M$. We see that $f(g h f) g=f g \in J(F ; k)$, which implies that $g h f \in$ $J(F ; k)$; that is, $(g h) f \neq 0$ in $P_{k}$. Then by Lemma 22, we get ( $g h) f \in R_{g h} \cap L_{f}$. Also, we have $g(h f) \in R_{g} \cap L_{h f}$. Whence ghf $\in L_{f} \cap R_{g}=L_{e} \cap R_{e}=H_{e}$, the group $\mathscr{\mathscr { H }}$-class contains $e$. So, there exists a positive integer $t$ such that $e=(g h f)^{t} \in M$ since $g, h, f \in M$. Therefore, $E(J(F ; k)) \subseteq M$. It follows from Lemma 27 that $Q(F ; k)=\langle E(J(F ; k))\rangle \subseteq M \subseteq Q(F ; k)$; hence $M=Q(F ; k)$, a contradiction. Therefore, $L_{\alpha} \cap M=\emptyset$ for some $\alpha \in J(F ; k)$ or $R_{\beta} \cap M=\emptyset$ for some $\beta \in J(F ; k)$. If $M \cap L_{\alpha}=\emptyset$ for some $\alpha \in J(F ; k)$, then

$$
M \subseteq Q(F ; k-1) \cup\left(J(F ; k) \backslash L_{\alpha}\right) \subsetneq Q(F ; k) .
$$

Thus, $M=Q(F ; k-1) \cup\left(J(F ; k) \backslash L_{\alpha}\right)$ by the maximality of $M$. If $M \cap R_{\beta}=\emptyset$ for some $\beta \in J(F ; k)$, then

$$
M \subseteq Q(F ; k-1) \cup\left(J(F ; k) \backslash R_{\beta}\right) \subsetneq Q(F ; k) .
$$

Thus, $M=Q(F ; k-1) \cup\left(J(F ; k) \backslash R_{\beta}\right)$ by the maximality of M.

Notice that the number of $\mathscr{L}$-classes in $J(F ; k)$ equals $\left(\begin{array}{l}r \\ k\end{array}\right)$ and the number of $\mathscr{R}$-classes in $J(F ; k)$ equals $S(r, k) k^{n-r}$ where $S(r, k)$ is the Stirling number of the second kind; see $[18$, Section 2] for details. Therefore, the number of maximal regular subsemigroups of $Q(F ; k)$ is equal to $\left(\begin{array}{l}r \\ k\end{array}\right)+S(r, k) k^{n-r}$ when $2 \leq k \leq r-1$. define

We shall normally write $T_{n}$ instead of $T(X)$. For $1 \leq k \leq n$,

$$
\begin{aligned}
J_{k} & =\left\{\alpha \in T_{n}:|X \alpha|=k\right\}, \\
K(n, k) & =\left\{\alpha \in T_{n}:|X \alpha| \leq k\right\} .
\end{aligned}
$$

It is well-known that $K(n, k)=J_{1} \cup J_{2} \cup \cdots \cup J_{k}$ and $K(n, k)$ is an ideal of $T_{n}$ for all $1 \leq k \leq n$. If $n=r$, then $F_{n, r}=$ $F_{n, n}=T_{n}, J(F ; k)=J_{k}$, and $Q(F ; k)=K(n, k)$. Therefore, we establish the following corollary which first appeared in [8, Theorem 2].

Corollary 33. Each maximal regular subsemigroup of $K(n, k)$, $2 \leq k \leq n-1$ must be one of the following forms: 
(1) $K(n, k-1) \cup\left(J_{k} \backslash L_{\alpha}\right)$;

(2) $K(n, k-1) \cup\left(J_{k} \backslash R_{\beta}\right)$,

where $\alpha, \beta \in J_{k}, L_{\alpha}$ is the $\mathscr{L}$-class containing $\alpha$ in $J_{k}$ of $T_{n}$, and $R_{\beta}$ is the $\mathscr{R}$-class containing $\beta$ in $J_{k}$ of $T_{n}$.

We note that if $n=r$, then we immediately obtain that the number of maximal regular subsemigroups of $K(n, k)$ is equal to $\left(\begin{array}{l}n \\ k\end{array}\right)+S(n, k)$ when $2 \leq k \leq n-1$.

\section{Conflicts of Interest}

The author declares that they have no conflicts of interest.

\section{Acknowledgments}

Financial support from the Coordinating Center for Thai Government Science and Technology Scholarship Students (CSTS), National Science and Technology Development Agency (NSTDA), is acknowledged.

\section{References}

[1] J. M. Howie, Fundamentals of Semigroup Theory, Oxford University Press, New York, NY, USA, 1995.

[2] B. M. Schein, "A symmetric semigroup of transformations is covered by its inverse subsemigroups," Acta Mathematica Hungarica, vol. 22, pp. 163-171, 1971/72.

[3] B. M. Schein, "Research problems," Semigroup Forum, vol. 1, pp. 91-92, 1970

[4] J. W. Nichols, "A class of maximal inverse subsemigroups of $T_{X}$," Semigroup Forum, vol. 13, no. 2, pp. 187-188, 1976.

[5] N. R. Reilly, "Maximal inverse subsemigroups of $T_{X}$," Semigroup Forum, vol. 15, no. 4, pp. 319-326, 1978.

[6] X. Yang, "A classification of maximal inverse subsemigroups of the finite symmetric inverse semigroups," Communications in Algebra, vol. 27, no. 8, pp. 4089-4096, 1999.

[7] X. Yang, "Maximal subsemigroups of the finite singular transformation semigroup," Communications in Algebra, vol. 29, no. 3, pp. 1175-1182, 2001.

[8] T. You, "Maximal regular subsemigroups of certain semigroups of transformations," Semigroup Forum, vol. 64, no. 3, pp. 391396, 2002.

[9] H. B. Yang and X. L. Yang, "Maximal subsemigroups of finite transformation semigroups $K(n, r)$," Acta Mathematica Sinica, vol. 20, no. 3, pp. 475-482, 2004.

[10] P. Zhao, H. Hu, and T. You, "A note on maximal regular subsemigroups of the finite transformation semigroups $T_{(n, r)}$," Semigroup Forum, vol. 88, no. 2, pp. 324-332, 2014.

[11] J. East, J. D. Mitchell, and Y. Péresse, "Maximal subsemigroups of the semigroup of all mappings on an infinite set," Transactions of the American Mathematical Society, vol. 367, no. 3, pp. 19111944, 2015.

[12] J. S. Symons, "Some results concerning a transformation semigroup," Journal of the Australian Mathematical Society: Pure Mathematics and Statistics. Series A, vol. 19, no. 4, pp. 413-425, 1975.

[13] S. Nenthein, P. Youngkhong, and Y. Kemprasit, "Regular elements of some transformation semigroups," Pure Mathematics and Applications, vol. 16, no. 3, pp. 307-314 (2006), 2005.
[14] J. Sanwong and W. Sommanee, "Regularity and Green's relations on a semigroup of transformations with restricted range," International Journal of Mathematics and Mathematical Sciences, vol. 2008, Article ID 794013, 11 pages, 2008.

[15] J. Sanwong, B. Singha, and R. P. Sullivan, "Maximal and minimal congruences on some semigroups," Acta Mathematica Sinica, vol. 25, no. 3, pp. 455-466, 2009.

[16] S. Mendes-Gonçalves and R. P. Sullivan, "The ideal structure of semigroups of transformations with restricted range," Bulletin of the Australian Mathematical Society, vol. 83, no. 2, pp. 289-300, 2011.

[17] J. Sanwong, "The regular part of a semigroup of transformations with restricted range," Semigroup Forum, vol. 83, no. 1, pp. 134146, 2011.

[18] W. Sommanee and J. Sanwong, "Rank and idempotent rank of finite full transformation semigroups with restricted range," Semigroup Forum, vol. 87, no. 1, pp. 230-242, 2013.

[19] V. H. Fernandes and J. Sanwong, "On the ranks of semigroups of transformations on a finite set with restricted range," Algebra Colloquium, vol. 21, no. 3, pp. 497-510, 2014.

[20] L. Sun and J. Sun, "A natural partial order on certain semigroups of transformations with restricted range," Semigroup Forum, vol. 92, no. 1, pp. 135-141, 2016.

[21] K. Tinpun and J. Koppitz, "Relative rank of the finite full transformation semigroup with restricted range," Acta Mathematica Universitatis Comenianae, vol. 85, no. 2, pp. 347-356, 2016.

[22] I. Dimitrova and J. Koppitz, "On the maximal regular subsemigroups of ideals of order-preserving or order-reversing transformations," Semigroup Forum, vol. 82, no. 1, pp. 172-180, 2011.

[23] W. Sommanee and J. Sanwong, "Order-preserving transformations with restricted range: regularity, Green's relations, and ideals," Algebra Universalis, vol. 74, no. 3-4, pp. 277-291, 2015.

[24] W. Sommanee and K. Sangkhanan, "The regular part of a semigroup of linear transformations with restricted range," Journal of the Australian Mathematical Society, vol. 103, no. 3, pp. 402-419, 2017. 


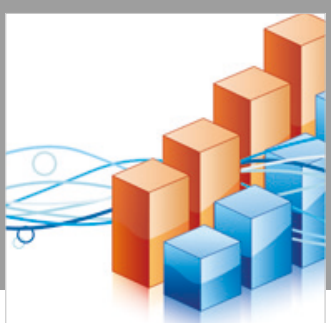

Advances in

Operations Research

\section{-n-m}
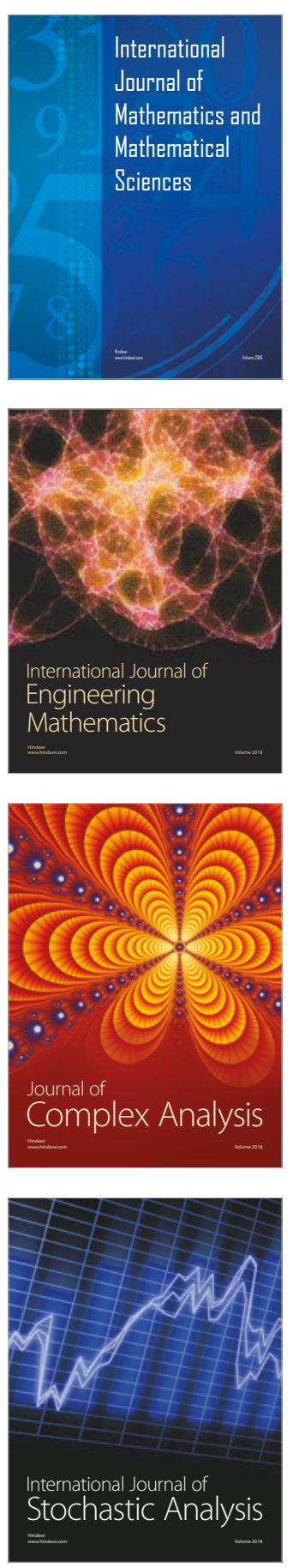
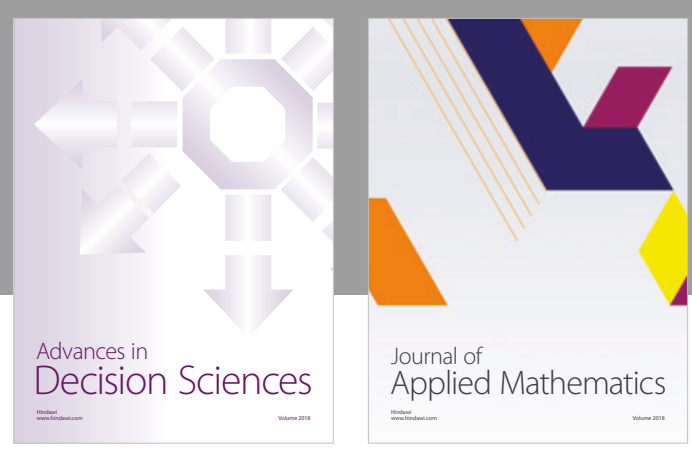

Journal of

Applied Mathematics
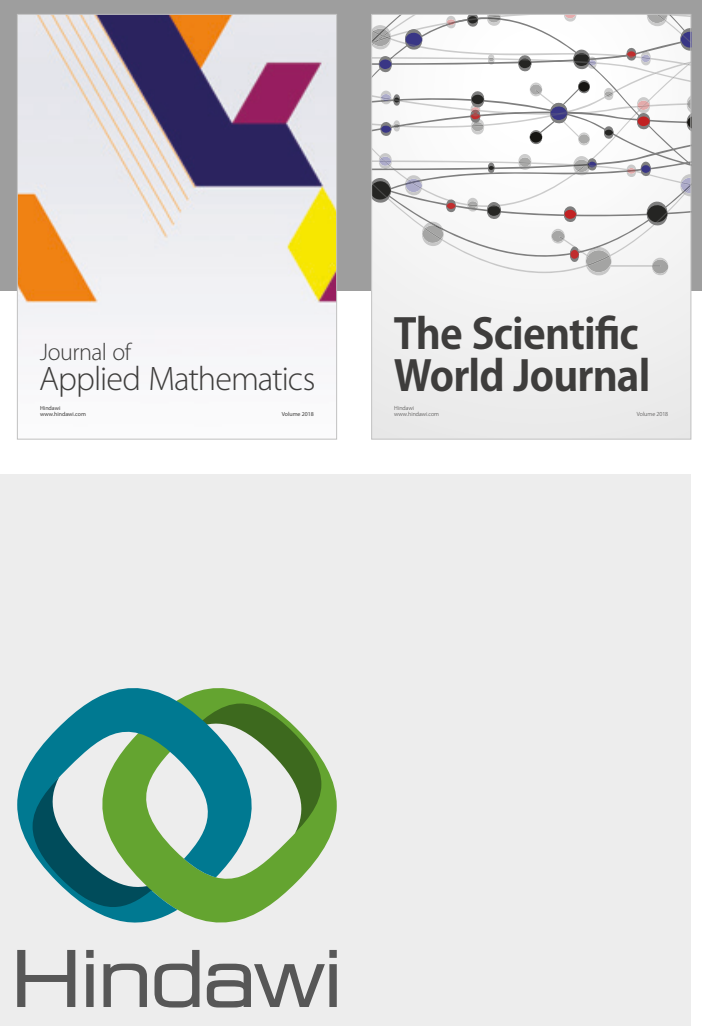

Submit your manuscripts at

www.hindawi.com

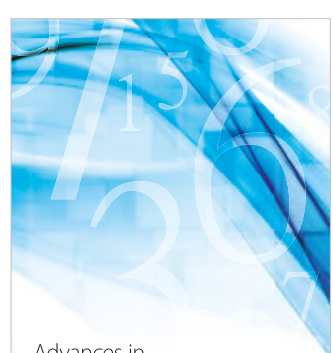

Advances in
Numerical Analysis
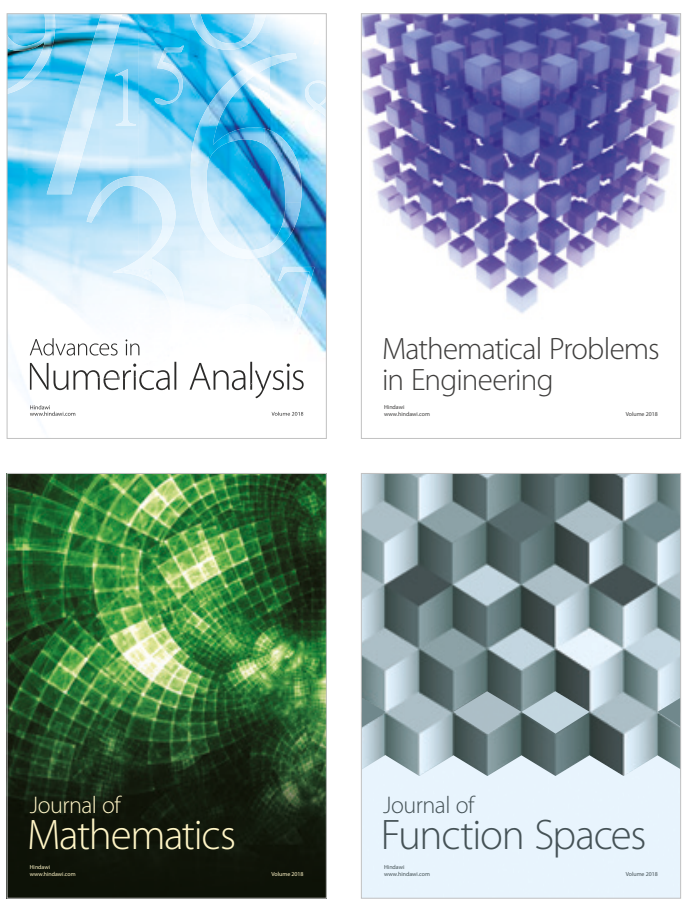

Mathematical Problems in Engineering

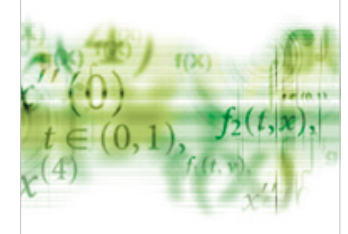

International Journal of

Differential Equations

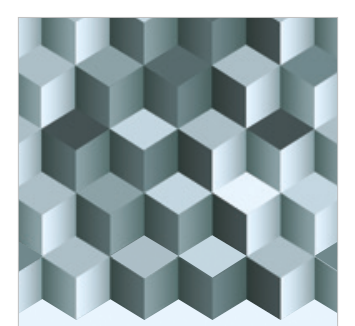

Journal of

Function Spaces

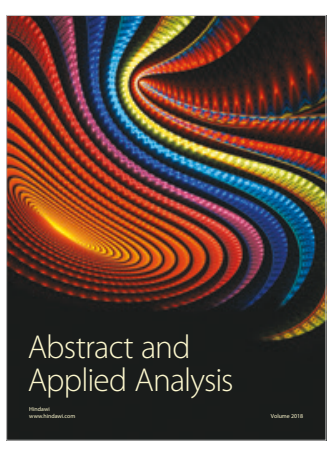

The Scientific

World Journal

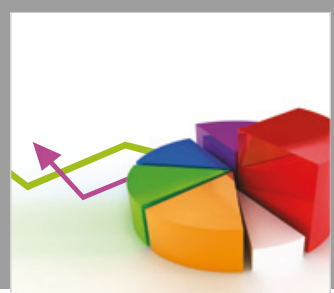

Journal of

Probability and Statistics
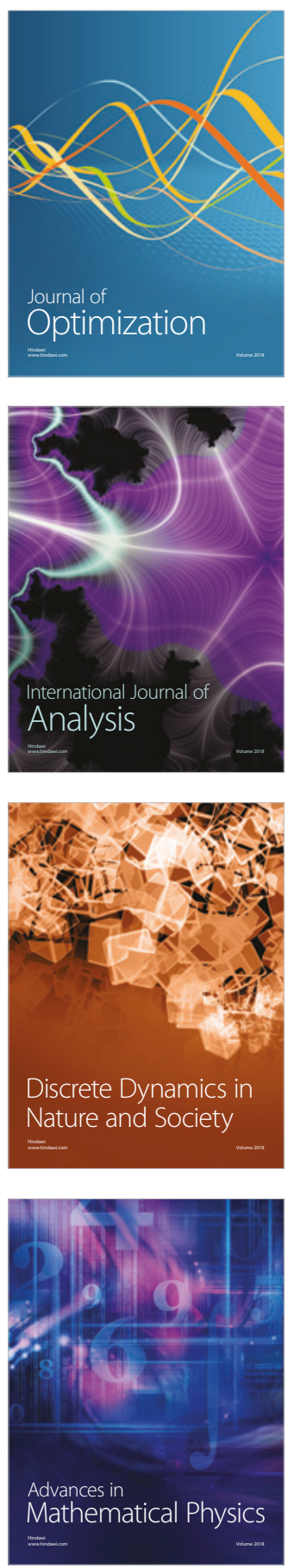